\title{
Multipartite unlockable bound entanglement in the stabilizer formalism
}

\author{
Guoming Wang 1 , $\rightarrow$ and Mingsheng Ying ${ }^{1,0}$ \\ ${ }^{1}$ State Key Laboratory of Intelligent Technology and Systems, \\ Department of Computer Science and Technology, Tsinghua University, Beijing, China, 100084
}

(Dated: September 25, 2018)

\begin{abstract}
We find an interesting relationship between multipartite bound entangled states and the stabilizer formalism. We prove that if a set of commuting operators from the generalized Pauli group on $n$ qudits satisfy certain constraints, then the maximally mixed state over the subspace stabilized by them is an unlockable bound entangled state. Moreover, the properties of this state, such as symmetry under permutations of parties, undistillability and unlockability, can be easily explained from the stabilizer formalism without tedious calculation. In particular, the four-qubit Smolin state [J. Smolin, Phys. Rev. A 63, 032306 (2001)] and its recent generalization to even number of qubits [S. Bandyopadhyay et al., Phys. Rev. A 71, 062317 (2005); R. Augusiak et al., Phys. Rev. A 73, 012318 (2006)] can be viewed as special examples of our results. Finally, we extend our results to arbitrary multipartite systems in which the dimensions of all parties may be different.
\end{abstract}

PACS numbers: 03.67.Mn

\section{INTRODUCTION}

As a peculiar phenomenon of quantum mechanics and a valuable resource for quantum information processing such as quantum computation [1], quantum cryptography [2], quantum teleportation [3] and superdense coding [4], entanglement has been extensively studied during the past years. One of the central problems about it is entanglement distillation [5], which is the procedure of extracting pure entangled states from many identical copies of a mixed entangled states by means of local operation and classical communication(LOCC). A surprising discovery in this area is that there exist mixed entangled states from which no pure entanglement can be distilled out, and these states are called bound entangled states [6]. Much effort has been devoted to the characterization and detection of bound entanglement $[6,7,8,9,10,11,12,13]$. Moreover, various properties and applications of bound entanglement have been found, including its irreversibility under LOCC manipulation 14], its capability of assisting the LOCC transformation of other entangled states [15] and distilling out classical secret bits [16], its violation of Bell inequalities [17, 18, 19], and so on [20, 21].

The distillability of multipartite entangled states, however, is much more complicated than that of bipartite entangled states. In the most natural case, we simply say that a multipartite entangled state is bound entangled if no pure entanglement can be distilled between any two parties by LOCC when all the parties remain spatially separated from each other. However, a multipartite bound entangled state may be 'unlocked' or 'activated' in the following sense: if we divide all the parties into several groups, and let each group join together and perform

\footnotetext{
*Electronic address: wgm00@mails.tsinghua.edu.cn
}

${ }^{\dagger}$ Electronic address: yingmsh@tsinghua.edu.cn collective quantum operations (or an equivalent way is to let them share a priori singlets, since they can use them to teleport their respective particles to a common party via quantum teleportation), then pure entanglement may be distilled between some two different groups. If so, this state is called an unlockable or activable bound entangled state.

There are two famous classes of multipartite unlockable bound entangled states that have been proposed. The first class includes a four-qubit state called the Smolin state 22 and its recent generalization to even number of qubits [23, 24]. These states have been applied in remote information concentration [20], quantum secret sharing [25], and reducing communication complexity [25, 26, 27]. Shor et al. also utilized the Smolin state to demonstrate a fascinating effect named 'superactivation' of bound entanglement [28, 29]. In addition, in 23] Bandyopadhyay et al. found that the Hilbert space of even number $(\geq 4)$ of qubits can always be decomposed as a direct sum of four orthogonal subspaces such that the normalized projectors onto the subspaces are activable bound entangled states. The other class, presented by Dür et al. [30, 31], has been used to demonstrate numerous possible ways in which bound entangled states can be activated. Besides, the relation between multipartite distillability and Bell inequalities was also studied in [12, 17, 18, 32]. Despite these progresses achieved, the general structure of multipartite unlockable bound entanglement still remains elusive.

The stabilizer formalism [33, 34], on the other hand, has also played a significant role in quantum information science, especially in quantum error correction codes [35, 36] and cluster state quantum computation [37. Its essential idea is to describe the quantum state by a set of stabilizing operators rather than the state vector. This formalism provides a very compact and effective way to describe and understand a lot of phenomena in quantum information.

In this paper we link the two seemingly irrelevant ar- 
eas and find an interesting relationship between them. In specific, we prove that if a set of commuting operators from the generalized Pauli group on $n$ qudits satisfy certain constraints, then the maximally mixed state over the subspace stabilized by them is an unlockable bound entangled state, and its properties can be easily explained from the stabilizer formalism. In particular, the Smolin state and its generalization are reinterpreted as one special case of our results. Furthermore, our results can also be extended to arbitrary multipartite systems in which the dimensions of all parties may be different.

This paper is organized as follows. In Sec. II we first briefly recall some facts about the generalized Pauli group and the stabilizer formalism, and then propose our main results. In Sec. III we analyze a series of examples by using our theorems. In Sev. IV, we extend our results to arbitrary multipartite systems. Finally, Sec. V summarizes our results.

\section{CONSTRUCTION OF MULTIPARTITE UNLOCKABLE BOUND ENTANGLED STATES}

\section{A. The generalized Pauli group and stabilizer formalism}

In this section we review some basic facts about the generalized Pauli group and the corresponding stabilizer formalism in the general high-dimensional case. Similar topics have also been explored in [38, 39, 40, 41].

Consider a $d$-dimensional Hilbert space. Define

$$
\begin{aligned}
& X_{(d)}=\sum_{j=0}^{d-1}|j \oplus 1\rangle\langle j|, \\
& Z_{(d)}=\sum_{j=0}^{d-1} \omega^{j}|j\rangle\langle j|,
\end{aligned}
$$

where $\omega=e^{i \frac{2 \pi}{d}}$ is the $d$-th root of unity over the complex field and the ' $\oplus$ ' sign denotes addition modulo $d$. Then the matrices $\left\{\sigma_{i, j}=X_{(d)}^{i} Z_{(d)}^{j}: i, j=0,1, \ldots, d-1\right\}$ are considered as the generalized Pauli matrices over the $d$-dimensional space, and they have the following commutation relation

$$
\sigma_{i, j} \sigma_{m, n}=\omega^{j m-i n} \sigma_{m, n} \sigma_{i, j} .
$$

It can be checked that when $d$ is odd, $\sigma_{i, j}$ always have eigenvalues $\left\{1, \omega^{c}, \omega^{2 c}, \ldots, \omega^{d-c}\right\}$ for some $c \mid d$ (i.e. $c$ is a factor of $d$ ); but when $d$ is even, the eigenvalues of $\sigma_{i, j}$ may be either of the above form or $\left\{\omega^{1 / 2}, \omega^{c+1 / 2}, \omega^{2 c+1 / 2}, \ldots, \omega^{d-c+1 / 2}\right\}$ for some $c \mid d$.

The generalized Pauli group on $n$ qudits $G_{n}$ is generated under multiplication by the Pauli matrices acting on each qudit, together with the phase factor $\gamma=\sqrt{\omega}$, i.e.

$$
\begin{aligned}
& G_{n}=\left\{\gamma^{a} \sigma_{i_{1}, j_{1}} \otimes \sigma_{i_{2}, j_{2}} \otimes \cdots \otimes \sigma_{i_{n}, j_{n}}: 0 \leq a \leq 2 d-1,\right. \\
& \left.0 \leq i_{1}, j_{1}, i_{2}, j_{2}, \ldots, i_{n}, j_{n} \leq d-1\right\} .
\end{aligned}
$$

Actually, when $d$ is odd, the introduction of $\gamma$ is unnecessary and it can be replaced by $\omega$ (For a detailed discussion about this, one can see [41]). However, this will not affect our results since in the following we consider only elements in $G_{n}^{\prime}=\left\{\bigotimes_{k=1}^{n} \sigma_{i_{k}, j_{k}}: \forall k=1,2, \ldots, n, i_{k}=0\right.$ or $\left.j_{k}=0\right\} \subset G_{n}$. For any element $g \in G_{n}^{\prime}$ it has eigenvalues $\left\{1, \omega^{c}, \omega^{2 c}, \ldots, \omega^{d-c}\right\}$ for some $c \mid d$.

Suppose we choose commuting operators $g_{1}, g_{2}, \ldots, g_{k}$ from $G_{n}^{\prime}$. Let $S=\left\langle g_{1}, g_{2}, \ldots, g_{k}\right\rangle$ denote the Abelian subgroup generated by them. A state $|\psi\rangle$ is said to be stabilized by $S$, or $S$ is the stabilizer of $|\psi\rangle$, if $g_{i}|\psi\rangle=$ $|\psi\rangle, \forall i=1,2, \ldots, k$. All the states stabilized by $S$ constitute a subspace denoted by $V_{S}$. With the fact that $\sum_{i=0}^{d-1} \omega^{c i}=0, \forall c=1,2, \ldots, d-1$, one can verify that the projection operator onto $V_{S}$ is

$$
P_{S}=\prod_{i=1}^{k} \frac{\left(I+g_{i}+g_{i}^{2}+\cdots+g_{i}^{d-1}\right)}{d},
$$

and the maximally mixed state over $V_{S}$ is $\rho_{S}=$ $P_{S} / \operatorname{tr}\left(P_{S}\right)$. In particular, if there is a unique pure state stabilized by $S$, i.e. $\operatorname{dim}\left(V_{S}\right)=1, g_{1}, g_{2}, \ldots, g_{k}$ are called a complete set of stabilizer generators and $S$ is called a complete stabilizer.

In practice we are often interested in the stabilized subspace $V_{S}$, which is the subspace spanned by the simultaneous eigenstates of the operators $\left\{g_{1}, g_{2}, \ldots, g_{k}\right\}$ with the eigenvalues $\{1,1, \ldots, 1\}$. But in general we can also consider the subspaces spanned by the simultaneous eigenstates of $\left\{g_{1}, g_{2}, \ldots, g_{k}\right\}$ corresponding to their other eigenvalues $\left\{\lambda_{1}, \lambda_{2}, \ldots, \lambda_{k}\right\}$, where $\lambda_{i}$ can be an arbitrary eigenvalue of $g_{i}$. All these subspaces have the same dimensions and form an orthogonal decomposition of the whole space. In particular, when $\left\{g_{1}, g_{2}, \ldots, g_{k}\right\}$ are a complete set of stabilizer generators, each of these subspaces is one-dimensional.

\section{B. Main results}

In the following, we define a partition of $\{1,2, \ldots, n\}$ to be a set of its proper subsets $\left\{T_{1}, T_{2}, \ldots, T_{m}\right\}$ such that $T_{i} \cap T_{j}=\emptyset, \forall i \neq j$ and $\cup_{i=1}^{m} T_{i}=\{1,2, \ldots, n\}$, and use $\left|T_{i}\right|$ to denote the number of elements in $T_{i}$. An $n$ qudit state $\rho^{12 \ldots n}$ is said to be separable with respect to a partition $\left\{T_{1}, T_{2}, \ldots, T_{m}\right\}$ if it can be written as

$$
\rho^{12 \ldots n}=\sum_{k} p_{k} \rho_{k}^{(1)} \otimes \rho_{k}^{(2)} \otimes \cdots \otimes \rho_{k}^{(m)}
$$

where $\sum_{k} p_{k}=1, p_{k}>0$ and $\rho_{k}^{(i)}$ is a density operator of the subsystem $T_{i}$.

In order to conveniently describe our results, we introduce the following definitions.

Definition 1 Suppose $g=\bigotimes_{k=1}^{n} \sigma_{i_{k}, j_{k}} \in G_{n}^{\prime}$. Then the restriction of $g$ on a subset $T \subset\{1,2, \ldots, n\}$ is defined as $g^{(T)}=\bigotimes_{k \in T} \sigma_{i_{k}, j_{k}}$. 
Definition 2 Two operators $g, h \in G_{n}^{\prime}$ are said to commute locally with respect to a partition $\left\{T_{1}, T_{2} \ldots, T_{m}\right\}$ of $\{1,2, \ldots, n\}$ if $g^{\left(T_{\alpha}\right)} h^{\left(T_{\alpha}\right)}=h^{\left(T_{\alpha}\right)} g^{\left(T_{\alpha}\right)}, \forall \alpha=1,2, \ldots, m$.

Definition 3 Suppose $g_{1}, g_{2}, \ldots, g_{k}$ are commuting elements in $G_{n}^{\prime} . \quad S=\left\langle g_{1}, g_{2}, \ldots, g_{k}\right\rangle$ is said to be separable with respect to a partition $\left\{T_{1}, T_{2}, \ldots, T_{m}\right\}$ of $\{1,2, \ldots, n\}$ if $g_{1}, g_{2} \ldots, g_{k}$ commute locally with respect to this partition. Otherwise, if such a partition does not exist, $S$ is said to be inseparable.

Note that in the third definition, the separability of a stabilizer with respect to any partition does not depend on the choice of its generators, so it is well-defined.

The following lemma establishes a connection between the separability of a stabilizer $S$ and the separability of the maximally mixed state over the stabilized subspace $V_{S}$ :

Lemma 1 Suppose $g_{1}, g_{2}, \ldots, g_{k}$ are commuting elements in $G_{n}^{\prime} . S=\left\langle g_{1}, g_{2} \ldots, g_{k}\right\rangle$ is separable with respect to a partition $\left\{T_{1}, T_{2} \ldots, T_{m}\right\}$ of $\{1,2, \ldots, n\}$ if and only if the maximally mixed state $\rho_{S}$ over the stabilized subspace $V_{S}$ is separable with respect to the same partition. So if $S$ is inseparable, then $\rho_{S}$ is a genuine n-qudit entangled state.

Proof:" $\Longrightarrow "$ : Suppose $S=\left\langle g_{1}, g_{2}, \ldots, g_{k}\right\rangle$ is separable with respect to a partition $\left\{T_{1}, T_{2}, \ldots, T_{m}\right\}$. Then for $\forall \alpha=1,2, \ldots, m$, the operators $g_{1}^{\left(T_{\alpha}\right)}, g_{2}^{\left(T_{\alpha}\right)}, \ldots, g_{k}^{\left(T_{\alpha}\right)}$ are mutually commutative and thus can be simultaneously diagonalized. Suppose $\left\{\left|\psi_{\beta_{\alpha}}^{(\alpha)}\right\rangle: \beta_{\alpha}=1,2, \ldots, d^{\left|T_{\alpha}\right|}\right\}$ are their simultaneous eigenstates corresponding to the eigenvalue $\lambda_{\beta_{\alpha}, j}^{\alpha}$ for each $j=1,2, \ldots, k$. Then it is obvious that the $n$-qudit states $\left|\psi_{\beta_{1}, \beta_{2}, \ldots, \beta_{m}}\right\rangle \equiv \bigotimes_{\alpha=1}^{m}\left|\psi_{\beta_{\alpha}}^{(\alpha)}\right\rangle$ are the simultaneous eigenstates of $\left\{g_{j}=\bigotimes_{\alpha=1}^{m} g_{j}^{\left(T_{\alpha}\right)}\right\}$ with the eigenvalue $\Pi_{\alpha=1}^{m} \lambda_{\beta_{\alpha}, j}^{\alpha}$ for each $j=1,2, \ldots, k$. They also form an orthonormal basis of the $n$-qudit space. In particular, let $P=\left\{\left(\beta_{1}, \beta_{2}, \ldots, \beta_{m}\right): \Pi_{\alpha=1}^{m} \lambda_{\beta_{\alpha}, j}^{\alpha}=\right.$ $1, \forall j=1,2, \ldots, k\}$. Then we have

$$
\rho_{S}=\frac{1}{|P|} \sum_{\left(\beta_{1}, \beta_{2}, \ldots, \beta_{m}\right) \in P} \bigotimes_{\alpha=1}^{m}\left|\psi_{\beta_{\alpha}}^{(\alpha)}\right\rangle\left\langle\psi_{\beta_{\alpha}}^{(\alpha)}\right|,
$$

which implies that $\rho_{S}$ is separable with respect to the partition $\left\{T_{1}, T_{2}, \ldots, T_{m}\right\}$.

"£": Suppose $\rho_{S}$ is separable with respect to the partition $\left\{T_{1}, T_{2}, \ldots, T_{m}\right\}$. Then there exists a state $|\psi\rangle \in V_{S}$ such that $|\psi\rangle$ can be written as $|\psi\rangle=$ $\bigotimes_{\alpha=1}^{m}\left|\psi^{(\alpha)}\right\rangle$, where $\left|\psi^{(\alpha)}\right\rangle$ is a state of the subsystem $T_{\alpha}$. Since $|\psi\rangle$ is stabilized by $S$, we have $|\psi\rangle=$ $g_{j}|\psi\rangle=\bigotimes_{\alpha=1}^{m} g_{j}^{\left(T_{\alpha}\right)}\left|\psi^{(\alpha)}\right\rangle, \forall j=1,2, \ldots, k$, which means that $\left|\psi^{(\alpha)}\right\rangle$ should be a simultaneous eigenstate of $g_{1}^{\left(T_{\alpha}\right)}, g_{2}^{\left(T_{\alpha}\right)}, \ldots, g_{k}^{\left(T_{\alpha}\right)}$ for each $\alpha=1,2, \ldots, m$. This is impossible if $g_{1}^{\left(T_{\alpha}\right)}, g_{2}^{\left(T_{\alpha}\right)}, \ldots, g_{k}^{\left(T_{\alpha}\right)}$ do not commute. To see this, we prove that any two elements $g, h \in G_{l}^{\prime}$ for any $l$ do not have a simultaneous eigenstate if $g, h$ do not commute. From Eq.(2) and Eq.(3) one can see that $g h=\omega^{f(g, h)} h g$ for some integer $f(g, h)$ determined by $g$ and $h$. If $g$ and $h$ do not commute, i.e. $\omega^{f(g, h)} \neq 1$, and they share a simultaneous eigenstate $|\psi\rangle$ which corresponds to the eigenvalues $\lambda, \mu$ of $g, h$ respectively, then we have

$$
\begin{aligned}
& g h|\psi\rangle=g(\mu|\psi\rangle)=\lambda \mu|\psi\rangle \\
& =\omega^{f(g, h)} h g|\psi\rangle=\omega^{f(g, h)} h(\lambda|\psi\rangle)=\omega^{f(g, h)} \mu \lambda|\psi\rangle,
\end{aligned}
$$

which implies that at least one of $\lambda$ and $\mu$ must be zero. But this contradicts with the fact that any operator in the generalized Pauli group has only nonzero eigenvalues. So $g_{1}, g_{2}, \ldots, g_{k}$ commute locally with respect to the partition $\left\{T_{1}, T_{2}, \ldots, T_{m}\right\}$ and $S=\left\langle g_{1}, g_{2}, \ldots, g_{k}\right\rangle$ is separable with respect to this partition.

With the help of Lemma 1, we find that the distillability and unlockability of $\rho_{S}$ generated by an incomplete stabilizer $S=\left\langle g_{1}, g_{2}, \ldots, g_{k}\right\rangle$ are determined by the separability of $S$, as the following theorem states:

Theorem 1 Suppose $g_{1}, g_{2}, \ldots, g_{k}$ are commuting elements in $G_{n}^{\prime}$. Let $S=\left\langle g_{1}, \ldots, g_{k}\right\rangle$. If

(1)for any $i \neq j \in\{1,2, \ldots, n\}$, there exits a partition $\left\{Q_{1}, Q_{2}, \ldots, Q_{m}\right\}$ with $i \in Q_{1}, j \in Q_{2}$ such that $S$ is separable with respect to this partition.

(2)there exists a partition $\left\{T_{1}, T_{2}, \ldots, T_{m}\right\}$ with $\left|T_{1}\right|>$ 1 such that $S$ is separable with respect to this partition and $S^{\left(T_{1}\right)}=\left\langle g_{1}^{\left(T_{1}\right)}, g_{2}^{\left(T_{1}\right)}, \ldots, g_{k}^{\left(T_{1}\right)}\right\rangle$ is an inseparable and complete stabilizer on $T_{1}$.

Then the maximally mixed state $\rho_{S}$ over the stabilized subspace $V_{S}$ is an unlockable bound entangled state. Moreover, for any partition $\left\{T_{1}, T_{2}, \ldots, T_{m}\right\}$ satisfying condition (2), pure entanglement among the parties inside $T_{1}$ can be distilled by letting the parties inside $T_{2}, T_{3}, \ldots, T_{m}$ join together respectively.

Proof: First, we prove that $\rho_{S}$ is undistillable. Consider any two parties $i, j \in\{1,2, \ldots, n\}$. By condition (1) and Lemma 1 we can find a partition $\left\{Q_{1}, Q_{2}, \ldots, Q_{m}\right\}$ with $i \in Q_{1}$ and $j \in Q_{2}$ such that $\rho_{S}$ is separable with respect to it. So it is impossible to distill out pure entanglement between $i$ and $j$, even between $Q_{1}$ and $Q_{2}$, by LOCC, as long as $Q_{1}$ and $Q_{2}$ remain spatially separated.

Next, we prove that $\rho_{S}$ can be unlocked. Consider the partition $\left\{T_{1}, T_{2}, \ldots, T_{m}\right\}$ which fulfills condition (2). Since $S$ is separable with respect to this partition, we can repeat exactly the same argument presented in the first part of the proof of Lemma 1 without changing any notations introduced. Now suppose all the parties inside $T_{\alpha}$ join together and perform the projection measurement in the basis $\left\{\left|\psi_{\beta_{\alpha}}^{(\alpha)}\right\rangle: \beta_{\alpha}=1, \ldots, d^{\left|T_{\alpha}\right|}\right\}$ for each $\alpha=2,3, \ldots, m$, and obtain the outcomes $\beta_{2}^{\prime}, \beta_{3}^{\prime}, \ldots, \beta_{m}^{\prime}$ respectively. Then by Eq.(6) we have the remaining state of the subsystem $T_{1}$ is

$$
\rho_{S}^{(1)}=\frac{1}{\left|P_{\beta_{2}^{\prime}, \beta_{3}^{\prime}, \ldots, \beta_{m}^{\prime}}\right|} \sum_{\beta_{1} \in P_{\beta_{2}^{\prime}, \beta_{3}^{\prime}, \ldots, \beta_{m}^{\prime}}}\left|\psi_{\beta_{1}}^{(1)}\right\rangle\left\langle\psi_{\beta_{1}}^{(1)}\right|,
$$


where $P_{\beta_{2}, \beta_{3}, \ldots, \beta_{m}}=\left\{\beta_{1}: \lambda_{\beta_{1}, j}^{1}=1 / \Pi_{\alpha=2}^{m} \lambda_{\beta_{\alpha}, j}^{\alpha}, \forall j=\right.$ $1,2, \ldots, k\}$. Since $S^{\left(T_{1}\right)}=\left\langle g_{1}^{\left(T_{1}\right)}, g_{2}^{\left(T_{1}\right)}, \ldots, g_{k}^{\left(T_{1}\right)}\right\rangle$ is a complete stabilizer on $T_{1}$, we have that $P\left(\beta_{2}^{\prime}, \beta_{3}^{\prime}, \ldots, \beta_{m}^{\prime}\right)$ actually contains only one element and therefore $\rho_{S}^{(1)}$ is a pure state. Moreover, because $S^{\left(T_{1}\right)}$ is inseparable, by Lemma 1 we know that $\rho_{S}^{(1)}$ is a genuine $\left|T_{1}\right|$-qudit entangled state. Therefore we have obtained an activation strategy.

Note that by a similar argument, we can easily prove that Lemma 1 and Theorem 1 will still hold if we replace $\rho_{S}$ by a maximally mixed state over the subspace spanned by the simultaneous eigenstates of $\left\{g_{1}, g_{2}, \ldots, g_{k}\right\}$ corresponding to their eigenvalues $\left\{\lambda_{1}, \lambda_{2}, \ldots, \lambda_{k}\right\}$, where $\lambda_{i}$ is an arbitrary eigenvalue of $g_{i}$. Recalling that all these subspaces have the same dimensions, we reach the following conclusion:

Theorem 2 Suppose $g_{1}, g_{2}, \ldots, g_{k}$ are $k$ commuting elements in $G_{n}^{\prime}$. If they satisfy the condition (1) and (2) in Theorem 1, and the subspace stabilized by them is bdimensional with $b \mid d^{n}$, then the Hilbert space of $n$ qudits can be decomposed into $\frac{d^{n}}{b}$ orthogonal subspaces such that the normalized projection operator onto each of them is an unlockable bound entangled state.

The two theorems above provide a simple method of constructing a wide class of unlockable bound entangled states in arbitrary multiqudit systems. What we need to do now is to appropriately choose several commuting operators from the generalized Pauli group on $n$ qudits. It is worth noting that our construction essentially utilizes the symmetry of the generalized Pauli matrices. Consequently the constructed states also own some inherent symmetry. With the help of Lemma 1, the properties of these states can be easily explained from the stabilizer formalism, as shown in the subsequent section.

\section{ILLUSTRATIONS}

In this section we will analyze several concrete examples by using our theorems. Without explicitly pointed out, the matrices $X$ and $Z$ appearing below are $X_{(d)}$ and $Z_{(d)}$ defined by Eq.(1) with the corresponding dimension $d$. We will also use the notation $X_{j}$ to denote the operation $X$ acting on the $j$ th party and similarly for $Z_{j}$.

Example 1: Consider a 4-qubit system. Define

$$
\begin{aligned}
& g_{1}=X_{1} X_{2} X_{3} X_{4}, \\
& g_{2}=Z_{1} Z_{2} Z_{3} Z_{4} .
\end{aligned}
$$

The maximally mixed state over the subspace stabilized by $g_{1}$ and $g_{2}$ is

$$
\rho^{(4)} \equiv \rho_{\left\langle g_{1}, g_{2}\right\rangle}=\frac{1}{16}\left(I+g_{1}\right)\left(I+g_{2}\right)
$$

Because $X \otimes X$ and $Z \otimes Z$ commute, $S=\left\langle g_{1}, g_{2}\right\rangle$ is separable with respect to any $2: 2$ partition of $\{1,2,3,4\}$, which assures that the condition (1) in Theorem 1 is fulfilled. Any $2: 2$ partition also satisfies the condition (2) in Theorem 1 since $S=\langle X \otimes X, Z \otimes Z\rangle$ is an inseparable and complete stabilizer on two qubits. So $\rho^{(4)}$ is an unlockable bound entangled state and pure entanglement can be distilled between any two parties.

Actually, this state is exactly the Smolin state which is originally defined as

$$
\rho^{(4)}=\frac{1}{4} \sum_{\alpha, \beta=0}^{1}\left|\Phi_{\alpha \beta}\right\rangle_{12}\left\langle\Phi_{\alpha \beta}|\otimes| \Phi_{\alpha \beta}\right\rangle_{34}\left\langle\Phi_{\alpha \beta}\right|,
$$

where

$$
\begin{aligned}
& \left|\Phi_{00}\right\rangle=\frac{1}{\sqrt{2}}(|00\rangle+|11\rangle), \quad\left|\Phi_{01}\right\rangle=\frac{1}{\sqrt{2}}(|00\rangle-|11\rangle), \\
& \left|\Phi_{10}\right\rangle=\frac{1}{\sqrt{2}}(|01\rangle+|10\rangle), \quad\left|\Phi_{11}\right\rangle=\frac{1}{\sqrt{2}}(|01\rangle-|10\rangle)
\end{aligned}
$$

are the four Bell states. To see this, one only need to realize that $\left|\Phi_{00}\right\rangle,\left|\Phi_{01}\right\rangle,\left|\Phi_{10}\right\rangle,\left|\Phi_{11}\right\rangle$ are the simultaneous eigenstates of $\{X \otimes X, Z \otimes Z\}$, with the eigenvalues $\{+1,+1\},\{-1,+1\},\{+1,-1\},\{-1,-1\}$, respectively. Considering the $2: 2$ partition $\{\{1,2\},\{3,4\}\}$, we have $g_{1}^{(\{1,2\})}=X_{1} X_{2}, g_{2}^{(\{1,2\})}=Z_{1} Z_{2}, g_{1}^{(\{3,4\})}=X_{3} X_{4}$ and $g_{2}^{(\{3,4\})}=Z_{3} Z_{4}$. So the four states $\left\{\left|\Phi_{\alpha \beta}\right\rangle_{12}\left|\Phi_{\alpha \beta}\right\rangle_{34}\right.$ : $\alpha, \beta=0,1\}$ are the simultaneous eigenstates of $g_{1}=$ $g_{1}^{(\{1,2\})} \otimes g_{1}^{(\{3,4\})}$ and $g_{2}=g_{2}^{(\{1,2\})} \otimes g_{2}^{(\{3,4\})}$ with the eigenvalues $\{1,1\}$. Thus by Eq. (6) $\rho^{(4)}$ can be written in the form of Eq.(11).

Furthermore, one can repeat the above argument by considering two other $2: 2$ partitions $\{\{1,3\},\{2,4\}\}$ and $\{\{1,4\},\{2,3\}\}$, and can easily concludes that $\rho^{(4)}$ can also be written as

$$
\begin{aligned}
\rho^{(4)} & =\frac{1}{4} \sum_{\alpha, \beta=0}^{1}\left|\Phi_{\alpha \beta}\right\rangle_{13}\left\langle\Phi_{\alpha \beta}|\otimes| \Phi_{\alpha \beta}\right\rangle_{24}\left\langle\Phi_{\alpha \beta}\right| \\
& =\frac{1}{4} \sum_{\alpha, \beta=0}^{1}\left|\Phi_{\alpha \beta}\right\rangle_{14}\left\langle\Phi_{\alpha \beta}|\otimes| \Phi_{\alpha \beta}\right\rangle_{23}\left\langle\Phi_{\alpha \beta}\right|
\end{aligned}
$$

which implies that $\rho^{(4)}$ is invariant under arbitrary permutation of the four parties. Note that this symmetry essentially arises from the fact that $g_{1}$ and $g_{2}$ both act identically on the four qubits.

By Eq.(11) and Eq.(13), $\rho^{(4)}$ is separable with respect to any $2: 2$ partition, and moreover, when any two parties get together and perform the projective measurement in the Bell basis, if their subsystem collapses into the state $\left|\Phi_{\alpha \beta}\right\rangle$, then the other two parties are in the same state $\left|\Phi_{\alpha \beta}\right\rangle$.

Example 2: Consider a system of $2 n(n \geq 2)$ qubits. Define

$$
\begin{aligned}
& g_{1}^{(2 n)}=X_{1} X_{2} X_{3} X_{4} \ldots X_{2 n-1} X_{2 n} \\
& g_{2}^{(2 n)}=Z_{1} Z_{2} Z_{3} Z_{4} \ldots Z_{2 n-1} Z_{2 n}
\end{aligned}
$$

Then the maximally mixed state over the subspace stabilized by $g_{1}^{(2 n)}$ and $g_{2}^{(2 n)}$ is

$$
\rho^{(2 n)} \equiv \rho_{\left\langle g_{1}^{(2 n)}, g_{2}^{(2 n)}\right\rangle}=\frac{1}{4^{n}}\left(I+g_{1}^{(2 n)}\right)\left(I+g_{2}^{(2 n)}\right) .
$$


One can easily check that $S=\left\langle g_{1}^{(2 n)}, g_{2}^{(2 n)}\right\rangle$ is separable with respect to any $2: 2: \cdots: 2$ partition of $\{1,2, \ldots, 2 n\}$, which ensures the satisfaction of condition (1) in Theorem 1. Moreover, any $2: 2: \cdots: 2$ partition satisfies the condition (2) in Theorem 1 . So $\rho^{(2 n)}$ is an unlockable bound entangled state and pure entangled state can be distilled between any two parties by letting the other $2 n-2$ parties pairwise group together.

Actually, $\rho^{(2 n)}$ is equivalent to the generalized Smolin state proposed in [23] and [24], up to an unimportant local Pauli operation. To see this, consider the $(2 n-2): 2$ partition $\{\{1,2, \ldots, 2 n-2\},\{2 n-1,2 n\}\}$. It is observed that $g_{1}^{(2 n)}, g_{2}^{(2 n)}$ commute locally with respect to this partition, and their restrictions on the subset $\{1,2, \ldots, 2 n-2\}$ are $g_{1}^{(2 n-2)}, g_{2}^{(2 n-2)}$ respectively. Let $\sigma_{00}=I_{1}, \sigma_{01}=Z_{1}, \sigma_{10}=X_{1}, \sigma_{11}=Y_{1}$ be the four Pauli operations acting on the first qubit. Then $\sigma_{\alpha \beta} \rho^{(2 n-2)} \sigma_{\alpha \beta}^{\dagger}$ is actually the maximally mixed state over the subspace spanned by the simultaneous eigenstate of $g_{1}^{(2 n-2)}, g_{2}^{(2 n-2)}$ with the eigenvalues $\left\{(-1)^{\beta},(-1)^{\alpha}\right\}$. Conseqently by Eq.(6) we have

$$
\rho^{(2 n)}=\frac{1}{4} \sum_{\alpha, \beta=0}^{1} \sigma_{\alpha \beta} \rho^{(2 n-2)} \sigma_{\alpha \beta}^{\dagger} \otimes\left|\Phi_{\alpha \beta}\right\rangle\left\langle\Phi_{\alpha \beta}\right|,
$$

which is the recursive definition of the generalized Smolin states in [23, 24] up to an local Pauli operation. Moreover, continuing this induction on $n$, one could at last get

$$
\rho^{(2 n)}=\frac{1}{4^{n-1}} \sum_{\oplus_{i=1}^{n} \alpha_{i}=\oplus_{i=1}^{n} \beta_{i}=0} \bigotimes_{i=1}^{n}\left|\Phi_{\alpha_{i} \beta_{i}}\right\rangle\left\langle\Phi_{\alpha_{i} \beta_{i}}\right|,
$$

where $\oplus$ denotes addition modulo 2. Noting that the two stabilizer generators $g_{1}^{(2 n)}$ and $g_{2}^{(2 n)}$ both act symmetrically on $2 n$ qubits, one can find that $\rho^{(2 n)}$ is invariant under arbitrary permutation of parties, which means Eq. (17) holds not only for the partition $\{\{1,2\},\{3,4\}, \ldots,\{2 n-1,2 n\}\}$ but also for arbitrary $2: 2: \cdots: 2$ partition.

Now suppose any $2 n-2$ parties join together pairwise and perform the projective measurement in the Bell basis. If the $n-1$ obtained outcomes are $\left|\Phi_{\alpha_{2}, \beta_{2}}\right\rangle,\left|\Phi_{\alpha_{3}, \beta_{3}}\right\rangle, \ldots,\left|\Phi_{\alpha_{n}, \beta_{n}}\right\rangle$ respectively, then by Eq.(17) the remaining two parties get one of the four Bell states $\left|\Phi_{\alpha_{1}, \beta_{1}}\right\rangle$ with $\alpha_{1}=\oplus_{i=2}^{n} \alpha_{i}$ and $\beta_{1}=\oplus_{i=2}^{n} \beta_{i}$.

In addition, by applying Theorem 2 to $g_{1}^{(2 n)}, g_{2}^{(2 n)}$ we know that the Hilbert space of $2 n(\geq 2)$ qubits can be decomposed into four orthogonal subspaces such that the normalized projection operator onto each of them is an unlockable bound entangled state, which was first pointed out in [23].

One may wonder whether there exists an analog of the Smolin state in systems of odd number of qubits. We believe that such a state is unlikely to exist, and even if it exists, it cannot be obtained by our method. Because if we want the constructed state to be symmetric under arbitrary permutation of parties, all the stabilizer generators should act equally on each qubit. But the tensor products of odd number of $X$ 's and $Z$ 's, or $X$ 's and $Y$ 's, or $Y$ 's and $Z$ 's, do not commute. Instead they anti-commute, e.g. $X^{\otimes 2 n+1} Z^{\otimes 2 n+1}=-Z^{\otimes 2 n+1} X^{\otimes 2 n+1}$. Therefore they cannot be simultaneously used as stabilizer generators.

From Example 1 and 2, we can see that the properties of the Smolin state and its generalization become so clear when they are redefined and reinterpreted in the stabilizer formalism. However, they are only two special instances which own the strongest symmetry. At the cost of losing symmetry to different extents, many more unlockable bound entangled states can be found in a similar way.

Example 3: Consider a 9-qubit system. Let

$$
\begin{aligned}
& g_{1}=X_{1} X_{2} Z_{3} X_{4} X_{5} Z_{6} X_{7} X_{8} Z_{9} \\
& g_{2}=X_{1} Z_{2} X_{3} X_{4} Z_{5} X_{6} X_{7} Z_{8} X_{9} \\
& g_{3}=Z_{1} X_{2} X_{3} Z_{4} X_{5} X_{6} Z_{7} X_{8} X_{9}
\end{aligned}
$$

The maximally mixed state over the subspace stabilized by them is

$$
\rho_{\left\langle g_{1}, g_{2}, g_{3}\right\rangle}=\frac{1}{2^{9}}\left(I+g_{1}\right)\left(I+g_{2}\right)\left(I+g_{3}\right) .
$$

The nine qubits of this state can be classified into three groups: $\{1,4,7\},\{2,5,8\}$, and $\{3,6,9\} . g_{1}, g_{2}$ and $g_{3}$ all act symmetrically on the three qubits of each group. So the state remains invariant when exchanging any two parties inside the same group. However, when exchange two parties that belongs to two different groups, such as 1 and 6 , the state will change.

Now consider two different partitions: $\quad\{\{1,2,3\},\{4,5,6\},\{7,8,9\}\} \quad$ and $\{\{1,6,8\},\{2,4,9\},\{3,5,7\}\}$. It can be verified that $S=\left\langle g_{1}, g_{2}, g_{3}\right\rangle$ is separable with respect to both of them and this fact ensures the satisfaction of the condition (1) in Theorem 1. Furthermore, the first partition $\{\{1,2,3\},\{4,5,6\},\{7,8,9\}\}$ also satisfies condition (2) in Theorem 1. Therefore, $\rho_{\left\langle g_{1}, g_{2}, g_{3}\right\rangle}$ is an unlockable bound entangled state and it can be unlocked as follows: let the parties 4, 5,6 join together and similarly for 7, 8, 9 . Then each of the two groups performs the projective measurement in the basis of the simultaneous eigenstates of three operators $\{X \otimes X \otimes Z, X \otimes Z \otimes X, Z \otimes X \otimes X\}$, then depending on their measurement outcomes a genuine three-qubit pure entangled state, which is also a simultaneous eigenstate of the three operators, is distilled out among the parties 1,2 and 3. In addition, by the symmetry of $\rho_{\left\langle g_{1}, g_{2}, g_{3}\right\rangle}$ presented above, we know that any three parties $i \in\{1,4,7\}, j \in\{2,5,8\}$ and $k \in\{3,6,9\}$ can obtain a genuine three-qubit pure entangled state among them by appropriately grouping the other six parties.

Example 4: Consider a 7-qutrit system, i.e. $d=3$. Let

$$
\begin{aligned}
& g_{1}=X_{1}^{2} Z_{2} Z_{3}^{2} X_{4} Z_{5}^{2} X_{6} Z_{7}, \\
& g_{2}=Z_{1} X_{2} X_{3}^{2} Z_{4} X_{5}^{2} Z_{6} X_{7}
\end{aligned}
$$


The maximally mixed state over the subspace stabilized by them is

$$
\rho_{\left\langle g_{1}, g_{2}\right\rangle}=\frac{1}{3^{7}}\left(I+g_{1}+g_{1}^{2}\right)\left(I+g_{2}+g_{2}^{2}\right) .
$$

The seven qutrits of this state can be classified into four groups: $\{1\},\{2,7\},\{3,5\}$ and $\{4,6\} . g_{1}, g_{2}$ both act symmetrically on the qutrits of each group. So the state remains invariant when exchanging any two parties inside the same group. However, it will vary when exchange two parties that belongs to two different groups, such as 1 and 2.

Consider two partitions: $\{\{1,2,3\},\{4,5\},\{6,7\}\}$ and $\{\{1,4\},\{2,5,7\},\{3,6\}\}$. It can be checked that $S=$ $\left\langle g_{1}, g_{2}\right\rangle$ is separable with respect to both of them, which makes the condition (1) in Theorem 1 fulfilled. Also, the partition $\{\{1,4\},\{2,5,7\},\{3,6\}\}$ satisfies condition (2) in Theorem 1. So $\rho_{\left\langle g_{1}, g_{2}\right\rangle}$ is an unlockable bound entangled state and can be activated in the following way: let the parties 2,5,7 join together and similarly for 3,6 . Then the first groups perform the projective measurement in the basis of the simultaneous eigenstates of the operators $\left\{Z_{2} Z_{5}^{2} Z_{7}, X_{2} X_{5}^{2} X_{7}\right\}$, and for the second group $\left\{Z_{3}^{2} X_{6}, X_{3}^{2} Z_{6}\right\}$. Depending on their measurement outcomes, a two-qutrit pure entangled state, which is one of the simultaneous eigenstates of $\left\{X_{1}^{2} X_{4}, Z_{1} Z_{4}\right\}$, is distilled out between the parties 1 and 4 .

Actually, one can verify that for any two parties $i \in$ $\{1,2,3,5,7\}$ and $j \in\{4,6\}$, a partition $\left\{\{i, j\}, T_{2}, T_{3}\right\}$ satisfying the condition (2) in Theorem 1 could be found, so $i$ and $j$ can share a two-qutrit pure entangled state by forming the groups $T_{2}$ and $T_{3}$. For example, for $i=2$ and $j=4$, such a partition is $\{\{2,4\},\{1,3,7\},\{5,6\}\}$.

To our knowledge, this state is the first presented unlockable bound entangled state in multiqutrit systems. Besides, by Theorem 2 we know that the Hilbert space of seven qutrits can be decomposed into nine orthogonal subspaces such that the normalized projection operator onto each of them is an unlockable bound entangled state.

In similar manners, numerous unlockable bound entangled states in arbitrary multiqudit systems can also be found. Moreover, one can similarly use our lemma and theorems to analyze the properties of these constructed states, such as symmetry under permutation of parties, separability and unlockability, from the stabilizer formalism.

\section{EXTENSION TO ARBITRARY MULTIPARTITE SYSTEMS}

In the previous sections, we considered only multiqudit systems. Actually, the distillability and unlockability of the constructed states $\rho_{S}$ depend mostly on the 'local commutation' relation of the stabilizer generators. The constraint that all parties should have the same dimensions is really unnecessary. Our definitions and theorems in Sec. II can be readily extended to arbitrary multipartite systems.

More precisely, consider a $d_{1} \times d_{2} \times \cdots \times d_{n}$ system where the $i$ th party has a $d_{i}$-dimensional space. Define $G^{\prime}\left(d_{1}, d_{2}, \ldots, d_{n}\right)=\left\{g: g=\bigotimes_{i=1}^{n} g_{i}\right.$ with $g_{i}=X_{\left(d_{i}\right)}^{a_{i}}$ or $Z_{\left(d_{i}\right)}^{b_{i}}$ for some $\left.a_{i}, b_{i}\right\}$. Then one can verify that for any element $g \in G^{\prime}\left(d_{1}, d_{2}, \ldots, d_{n}\right)$, its eigenvalues are in the form $\left\{1, \omega^{c}, \omega^{2 c}, \ldots, \omega^{D-c}\right\}$, where $\omega=e^{i \frac{2 \pi}{D}}, D$ is the least common multiple of $d_{1}, d_{2}, \ldots, d_{n}$, and $c \mid D$.

Suppose we choose commuting elements $g_{1}, g_{2}, \ldots, g_{k}$ from $G^{\prime}\left(d_{1}, d_{2}, \ldots, d_{n}\right)$. Let $S=\left\langle g_{1}, g_{2}, \ldots, g_{k}\right\rangle$ denote the Abelian group generated by them. Still we use $V_{S}$ to denote the subspace stabilized by $S$. Then with the fact that $\sum_{i=0}^{D-1} \omega^{c i}=0, \forall c=1,2, \ldots, D-1$, one can see that the projection operator onto $V_{S}$ is given by

$$
P_{S}=\prod_{i=1}^{k} \frac{\left(I+g_{i}+g_{i}^{2}+\cdots+g_{i}^{D-1}\right)}{D} .
$$

And the maximally mixed state over $V_{S}$ is $\rho_{S}=$ $P_{S} / \operatorname{tr}\left(P_{S}\right)$. Then following the same route of Sec.II.B, we can generalize the three definitions and the Lemma 1, Theorem 1, Theorem 2 to the elements in $G^{\prime}\left(d_{1}, d_{2}, \ldots, d_{n}\right)$.

Next we would like to use an example to illustrate this general case. Consider a $2 \times 2 \times 4 \times 4 \times 6 \times 6$ system. Let

$$
\begin{aligned}
& g_{1}=X_{(2)} \otimes Z_{(2)} \otimes X_{(4)}^{2} \otimes Z_{(4)} \otimes X_{(6)}^{3} \otimes Z_{(6)}, \\
& g_{2}=Z_{(2)} \otimes X_{(2)} \otimes Z_{(4)} \otimes X_{(4)}^{2} \otimes Z_{(6)} \otimes X_{(6)}^{3} .
\end{aligned}
$$

where $X_{(d)}, Z_{(d)}$ are defined as Eq.(1). $g_{1}$ and $g_{2}$ both have eigenvalues $1, \omega, \omega^{2}, \ldots, \omega^{11}$ where $\omega=e^{i \frac{\pi}{6}}$. The maximally mixed state over the subspace stabilized by $g_{1}, g_{2}$ is

$$
\rho_{\left\langle g_{1}, g_{2}\right\rangle}=\frac{1}{N}\left(\sum_{i=0}^{11} g_{1}^{i}\right)\left(\sum_{j=0}^{11} g_{2}^{j}\right),
$$

where $N=2 \times 2 \times 4 \times 4 \times 6 \times 6$ is the dimension of the whole space.

One can verify that $S=\left\langle g_{1}, g_{2}\right\rangle$ is separable with respect to any $2: 2: 2$ partition, e.g. $\{\{1,2\},\{3,4\},\{5,6\}\}$. So this state is separable with respect to any $2: 2: 2$ partition. In addition, any two parties can obtain a pure entangled state by letting the other four parties join together pairwise in an arbitrary fashion. This is because, as one may check, any $2: 2: 2$ partition satisfies the condition (2) in Theorem 1. For instance, consider the partition $\{\{1,6\},\{2,3\},\{4,5\}\}$. Suppose the parties 2 and 3 join together, and similarly for 4 and 5 . If the group $\{2,3\}$ perform the projective measurement in the basis of the simultaneous eigenstates of $\left\{Z_{(2)} \otimes X_{(4)}^{2}, X_{(2)} \otimes Z_{(4)}\right\}$, and the group $\{4,5\}$ perform the projective measurement in the basis of the simultaneous eigenstates of $\left\{Z_{(4)} \otimes X_{(6)}^{3}, X_{(4)}^{2} \otimes Z_{(6)}\right\}$, then depending on their outcomes, a pure entangles state, which is a simultaneous 
eigenstate of $\left\{X_{(2)} \otimes Z_{(6)}, Z_{(2)} \otimes X_{(6)}^{3}\right\}$, will be obtained between 1 and 6 .

It is worth noting that in this example although the six particles have three different kinds of dimensions 2, 4, 6 , as shown above, the unlockability of this state is very strong. So we learn that the distinction between the dimensions of different parties is not really an obstacle of building unlockable bound entangled states in such systems. Nonetheless, we should point out that the conditions in Theorem 1 may be not satisfiable for some multipartite systems. One instance is the multipartite system in which the dimensions of all parties are mutually relative prime. But what we guarantee is that when the conditions in Theorem 1 are fulfilled, we can use the theorem to build a class of unlockable bound entangled states in the corresponding multipartite system.

\section{CONCLUSION}

In sum, we find an interesting relationship between two important areas in quantum information science - multipartite bound entangled states and the stabilizer formalism. Our results provide a simple way of constructing unlockable bound entangled states in arbitrary multiqudit systems. These states not only can be concisely described, but also possess properties which can be easily explained from the stabilizer formalism. In particular, the previous four-qubit Smolin states and its generalization to even number of qubits can be viewed as special examples of our results. Our theorems can also be extended to arbitrary multipartite systems in which the dimensions of all parties may be different, although their conditions may be in fact unsatisfiable in some cases.

Finally, we would like to point out several directions for further investigation along our way. The first one would be to extend our work to more general situations. In our work we utilized the inherent symmetry of Pauli matrices to construct our unlockable bound entangled states. However, as the reader may have already found out, our construction actually mainly relies on the 'local commutation' relation of the stabilizer generators. This relation can be also defined over arbitrary multipartite operations which can be written as the tensor products of unitary operations on each subsystem, not just the generalized Pauli operations. Therefore it is entirely possible that our definitions and theorems can be appropriately adjusted so that it can be applicable to a wider class of multipartite operations and states. Another direction would be to study the properties and applications of our constructed unlockable bound entangled stated, such as their violation of Bell inequalities, whether they also show the 'superactivation' phenomenon, whether they can be used in the information processing tasks such as remote information concentration and multipartite key distribution. We hope that in this way more interesting results about the structures and features of multipartite bound entanglement will be found in the future.

\section{Acknowledgement}

We would like to thank Runyao Duan, Zhengfeng Ji, Yuan Feng and Chi Zhang for helpful discussions. This work was partly supported by the Natural Science Foundation of China (Grant Nos. 60621062 and 60503001) and the Hi-Tech Research and Development Program of China (863 project) (Grant No. 2006AA01Z102).
[1] P.W. Shor, in Proceedings of the 35th Annual Symposium on Foundations of Computer Science (IEEE Computer Society Press, Los Alamos, CA, 1994), p. 124.

[2] C. H. Bennett and G. Brassard, in Proceedings of the IEEE International Conference on Computers, Systems and Signal Processing, Bangalore IEEE, Piscataway, NJ, 1984, pp. 175-179; A. K. Ekert, Phys. Rev. Lett. 67, 661 (1991).

[3] C. H. Bennett, G. Brassard, C. Crepeau, R. Jozsa, A. Peres, and W. K. Wootters, Phys. Rev. Lett. 70, 1895 (1993).

[4] C. H. Bennett and S. J. Wiesner, Phys. Rev. Lett. 69, 2881 (1992).

[5] C. H. Bennett, G. Brassard, S. Popescu, B. Schumacher, J. A. Smolin, and W. K. Wootters, Phys. Rev. Lett. 76, 722 (1996); C. H. Bennett, H. J. Bernstein, S. Popescu, and B. Schumacher, Phys. Rev. A 53, 2046 (1996).

[6] M. Horodecki, P. Horodecki and R. Horodecki, Phys. Rev. Lett. 80, 5239 (1998).

[7] A. Peres, Phys. Rev. Lett. 77, 1413 (1996).

[8] P. Horodecki, M. Horodecki and R. Horodecki, Phys. Rev. Lett. 82, 1056 (1999).

[9] C.H. Bennett, D.P. DiVincenzo, Tal Mor, P.W. Shor,
J.A. Smolin, and B.M. Terhal, Phys. Rev. Lett. 82, 5385 (1999).

[10] P. W. Shor, J. A. Smolin and B. M. Terhal, Phys. Rev. Lett. 86, 2681 (2001).

[11] T. Hiroshima, Phys. Rev. Lett. 91, 057902 (2003).

[12] L. Masanes, Phys. Rev. Lett. 97, 050503 (2006).

[13] W. Dür, J. I. Cirac, M. Lewenstein and D. Bruß, Phys. Rev. A 61, 062313 (2000).

[14] D. Yang, M. Horodecki, R. Horodecki and B. SynakRadtke, Phys. Rev. Lett. 95, 190501 (2005).

[15] S. Ishizaka, Phys. Rev. Lett. 93, 190501 (2004).

[16] K. Horodecki, M. Horodecki, P. Horodecki and J. Oppenheim, Phys. Rev. Lett. 94, 160502 (2005).

[17] W. Dür, Phys. Rev. Lett. 87, 230402 (2001).

[18] A. Acín, Phys. Rev. Lett. 88, 027901 (2002).

[19] R. Augusiak and P. Horodecki, Phys. Rev. A 74, 010305(R) (2006).

[20] M. Murao and V. Vedral, Phys. Rev. Lett. 86, 352 (2001).

[21] L. Masanes, Phys. Rev. Lett. 96, 150501 (2006).

[22] J. A. Smolin, Phys. Rev. A 63, 032306 (2001).

[23] S. Bandyopadhyay, I. Chattopadhyay, V. Roychowdhury and D. Sarkar, Phys. Rev. A 71, 062317 (2005).

[24] R. Augusiak and P. Horodecki, Phys. Rev. A 73, 012318 
(2006).

[25] R. Augusiak and P. Horodecki, Phys. Rev. A. 74, 010305(R) (2006).

[26] C. Brukner, M. Żukowski, and A. Zeilinger, Phys. Rev. Lett. 89, 197901 (2002).

[27] C. Brukner, M. Żukowski, J.-W. Pan, and A. Zeilinger, Phys. Rev. Lett. 92, 127901 (2004).

[28] P. W. Shor, J. A. Smolin and A. V. Thapliyal, Phys. Rev. Lett 90, 107901 (2003).

[29] S. Bandyopadhyay and V. Roychowdhury, Phys. Rev. A 72, 060303(R) (2005).

[30] W. Dür, J.I. Cirac, and R. Tarrach, Phys. Rev. Lett. 83, 3562 (1999).

[31] W. Dür and J. I. Cirac, Phys. Rev. A 62, 022302 (2000).

[32] A. Acín, V. Scarani and M. M. Wolf, Phys. Rev. A 66, 042323 (2002).
[33] D. Gottesman, Phys. Rev. A 54, 1862 (1996).

[34] D. Gottesman, Ph.D. thesis, California Institute of Technology, Pasadena, CA, 1997.

[35] P.W. Shor, Phys. Rev. A 52, R2493 (1995).

[36] A. M. Steane, Phys. Rev. A 54, 4741 (1996).

[37] R. Raussendorf and H. J. Briegel, Phys. Rev. Lett. 86, 5188 (2001).

[38] D. Gottesman, in Quantum Computing and Quantum Communications: First NASA International Conference, edited by C. P. Williams Springer-Verlag, Berlin, 1999.

[39] A. Y. Vlasov, e-print quant-ph/0210049.

[40] M. A. Nielsen, M. J. Bremner, J. L. Dodd, A. M. Childs, and C. M. Dawson, Phys. Rev. A 66, 022317 (2002).

[41] E. Hostens, J. Dehaene and B. De Moor, Phys. Rev. A 71, 042315 (2005). 